\title{
A UTILIZAÇÃO DE MAPAS COGNITIVOS PARA ESTRUTURAÇÃO DO SISTEMA DE AUDITORIA AMBIENTAL PORTUÁRIA
}

\section{STRUCUTURING HARBOUR ENVIRONMENTAL AUDITORY SYSTEM USING COGNITIVE MAPS}

\author{
Moacyr Machado Cardoso Junior* E-mail: moacyr@ita.br \\ Armando Gonçalves Madeira Junior* E-mail: \\ madeira ita@yahoo.com.br \\ Carmen Belderrain* E-mail: carmen@ita.br \\ * ITA, São José dos Campos, SP
}

\begin{abstract}
Resumo: Este trabalho propõe estruturar um sistema de auditoria ambiental de portos de forma a minimizar os impactos ambientais e acidentes, proporcionando o bem estar dos trabalhadores e da comunidade local. Para atingir este objetivo deste problema interdisciplinar complexo, foi aplicada a técnica de mapas cognitivos para obtenção de uma árvore hierárquica para posterior utilização de outra metodologia, como uma de abordagem de critério único de síntese.
\end{abstract}

Palavras-chave: Decisão Multicriterial. Desempenho Portuário. Estruturação de Problemas. Gestão Ambiental. Mapas Cognitivos.

Abstract: This study proposes to structure a harbour environmental auditory system in order to minimize environmental and accidental impacts, providing employees and local community wellbeing. To achieve the main purpose of this paper, which is an interdisciplinary complex problem, was applied the cognitive maps technique to obtain a hierarchical tree to be utilized by other methodology, as an unique synthesis criterion approach.

Key-words: Cognitive Maps. Environmental Management. Harbour Performance. Multicriteria Decision. Problem Structuring.

\section{INTRODUÇÃO}

Os portos são elementos de vital importância para o fluxo de comércio exterior e interno. Suas infraestruturas são portais de entrada e saída de mercadorias para o modal marítimo que via de regra apresenta menor custo operacional, atributo essencial para a logística das empresas em um mercado cada vez mais globalizado. 
Atualmente, a avaliação de desempenho de serviços de transporte de carga não mais considera apenas a componente operacional, está transcendendo ao mercado "considerando os impactos sociais e ambientais que a organização gera no meio em que está inserida" (CAIXETA-FILHO, 2001).

A necessidade de auditorias ambientais está positivada no art. $33, \S 1^{\circ}$, VII da Lei 8.630/93, passível de fiscalização pelo órgão regulatório (ANTAQ, 2009). A Resolução CONAMA $n^{\circ} 237$ dispõe sobre a obrigatoriedade da realização de medidas de controle ambiental para renovação periódica da licença de Operação Portuária e a Resolução CONAMA n 306 que estabelece a avaliação dos sistemas de gestão e controle ambiental nos portos por auditores independentes.

Diante desta tendência normativa é importante que a autoridade portuária realize auditorias no setor ambiental e de segurança de forma a minimizar efeitos indesejados da exploração da atividade portuária.

A estruturação de um sistema de auditoria ambiental portuária é um problema interdisciplinar e pode ser classificado segundo MINGERS; ROSENHEAD, (2004) como problema não estruturado pela existência de pelo menos um dos fatores: múltiplos atores, perspectivas, conflitos de interesses, importâncias intangíveis e incertezas.

Para abordar este tipo de problema é mais adequado adotar um método que considere uma visão soft da pesquisa operacional (PO), onde a idéia do problema é deslocada para a percepção do observador (natureza subjetivista) segundo o paradigma do construtivismo (ENSSLIN et al 2005).

Strategic Options Development and Analysis - SODA é uma abordagem desenvolvida para auxiliar consultores, ou facilitadores, a ajudar seus clientes envolvidos com problemas não estruturados segundo a visão soft da PO. Dentro desta abordagem, os mapas cognitivos são a principal ferramenta para a estruturação dos problemas em questão. (ROSENHEAD; MINGERS, 2001).

A Segurança Portuária vem ganhando destaque nos últimos anos, a partir da implementação da Norma Regulamentadora NR-29, mas ainda carece de muitas melhorias. O maior entrave na aplicação da Norma continua sendo a forma de gestão da segurança na área portuária. As administrações dos portos estão voltadas 
para o embarque e desembarque no menor tempo possível, prevalecendo a lógica da Produção em detrimento da Proteção, conforme relatado por VIASEG, (2008).

O artigo está estruturado da seguinte forma: após esta seção, de natureza introdutória, a seção 2 aborda o Método de construção do Mapa Cognitivo com uma breve revisão sobre o tema, apresenta a ferramenta mapas cognitivos, sua construção e análise visando a criação da árvore hierárquica; a seção 3 dispõe sobre os resultados obtidos após a aplicação da técnica estudada na seção anterior no ambiente portuário; e a última seção apresenta reflexões finais e faz recomendações para pesquisas futuras.

O trabalho buscará contribuir para a estruturação de um problema complexo interdisciplinar de vital importância para o Brasil, visto que cerca de $98 \%$ do comércio exterior circula por meios dos portos cuja atividade é potencialmente causadora de impactos ambientais, focalizando a passagem do mapa cognitivo para a árvore hierárquica.

A estruturação tem por objetivo a descrição completa do problema tratado neste artigo, sendo classificada como uma problemática de descrição (P.ঠ), ou seja, definir os aspectos essenciais segundo o Decisor a serem levados em consideração no processo futuro de tomada de decisão (ENSSLIN; MONTIBELLER; NORONHA, 2001).

A abordagem metodológica foi a da pesquisa exploratória, permeada pela lógica dedutiva e indutiva, aplicadas de forma alternada, por ser uma característica própria dos Mapas Cognitivos. Por ser um problema complexo não estruturado assume-se a hipótese que a solução do problema é única e dependente do sistema de valores do decisor que participou do processo da estruturação.

\section{MÉTODO}

\subsection{Mapas Cognitivos}

Ensslin; Montibeller e Noronha (2001) caracteriza problema como uma situação onde o decisor deseja que alguma coisa seja diferente de como ela é e não está seguro de como obtê-la. O mapa cognitivo é uma técnica para que o decisor ou grupo de decisores consiga representar seu próprio problema. 
O mapa cognitivo é uma representação gráfica de um conjunto de representações discursivas feitas por alguma pessoa (decisor) referente a um objeto em um contexto de uma interação particular, DINIZ \& LINS, (2009). Pode ser definido como uma representação cognitiva quádrupla, defasado no tempo Ensslin; Montibeller e Noronha (2001), conforme dispõe a Figura 1.

Figura 1 - Modelo de construção do Mapa Cognitivo.

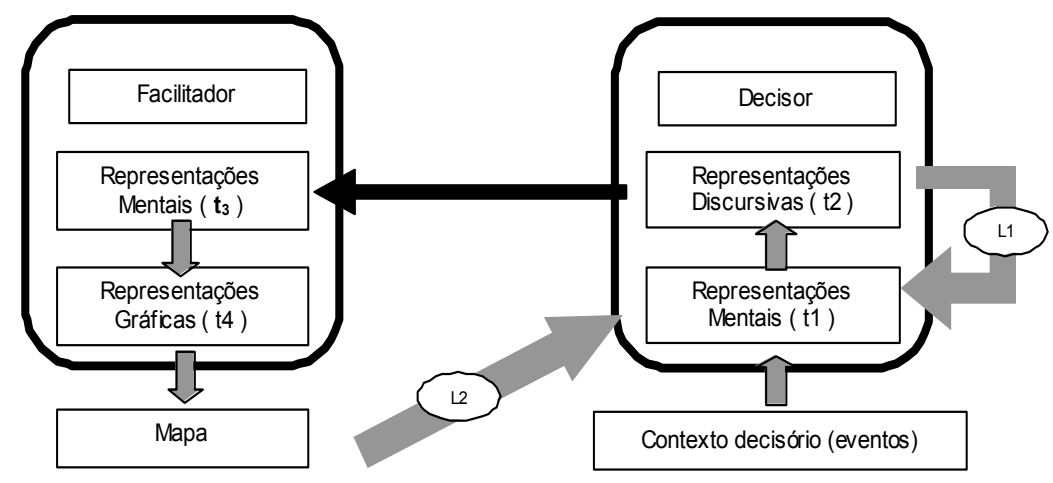

Fonte: (ENSSLIN, et al., 2001)

É quádrupla e defasada no tempo porque no momento $\mathrm{t} 1$ há representações mentais por parte do decisor que se projeta no instante t2 em representações discursivas (oral ou escrito) que serão passadas ao facilitador por meio do discurso.

O facilitador interpreta este discurso em t3 gerando suas representações mentais, para que em t4 as transforme em representação gráfica por meio do mapa cognitivo. Existe uma realimentação entre decisor e facilitador pela seta L2, o que reduz o hiato entre a representação mental do decisor e a construção do mapa cognitivo. Está presente o paradigma do construtivismo e aprendizagem pelas setas L1 e L2 onde o decisor desenvolve o problema tanto com o mapa cognitivo quanto este descreve o problema ao facilitador pelo discurso.

Existem diversos tipos de mapas cognitivos, porém será alvo do artigo apenas os mapas causais ou de influência por serem mais adequados ao auxílio à estruturação de modelos multicritério, segundo Ensslin, Montibeller e Noronha (2001). 


\subsection{Construção do Mapa Cognitivo}

Antes de construir o mapa cognitivo é de vital importância definir, dentre os diversos atores do processo decisório, quais pessoas (stakeholders) são representativas para a estruturação do problema, bem como seu grau de influência na tomada de decisão, para que estas mais relevantes participem da construção dos mapas cognitivos individuais, a formação do mapa cognitivo agregado e do congregado.

Ensslin, Montibeller e Noronha (2001), definem as seguintes etapas para a construção de um mapa cognitivo:

1. Definição de um rótulo para o problema;

2. Definição dos elementos primários de avaliação (EPA);

3. Construção de conceitos (e seu pólo oposto, para mapas bipolares) a partir dos EPA;

4. Construção da hierarquia de conceitos (fins, meios e atributos); e

5. Estabelecimento das ligações de influência (positivas e negativas).

\subsection{Análise de Mapas Cognitivos}

A análise do mapa cognitivo realizada pelo facilitador visa, por intermédio deste, estruturar o modelo multicritério. Consiste em definir quais são os aspectos, dentro do contexto decisório, que o decisor considera essenciais e desejáveis de serem levados em conta no processo e avaliação. Para tal existem dois tipos de análise: a tradicional que considera fundamentalmente a forma do mapa; e a avançada que leva em conta não só a forma como também o conteúdo dos conceitos. (ENSSLIN; MONTIBELLER; NORONHA, 2001).

A análise tradicional verifica inicialmente a estrutura hierárquica do mapa cognitivo (meios e fins), identificando conceitos cabeças, os quais chegam as flechas, que revelam objetivos; e conceitos rabos, de onde saem as flechas, que se trata de ações ou alternativas através dos quais podem atingir os objetivos dos decisores. 
Encerra este tipo de análise com o agrupamento do mapa. O cluster é definido como um conjunto de nós que são relacionados por ligações intra-componentes fortes que se relaciona com outros grupos do mapa cognitivo por ligações mais fracas (ligações inter-componentes).

A detecção dos grupos permite que o facilitador analise cada um deles de forma independente, reduzindo a complexidade do mapa cognitivo.

Encerrada o agrupamento da análise tradicional, parte-se para a análise avançada que consiste basicamente de identificação das linhas de argumentação, observando a forma do mapa; e, posteriormente, com a análise de conteúdo destas linhas, estas são agrupadas em ramos.

A linha de argumentação se constitui por uma cadeia de conceitos que são influenciados e hierarquicamente superiores a um conceito rabo, inicia-se em um conceito rabo e encerra em um conceito cabeça. As linhas de argumentação são chamadas de internas quando começa num conceito rabo e termina no conceito cabeça dentro do mesmo cluster.

Analisando o conteúdo das linhas de argumentação, são agrupadas em ramos as linhas que demonstrem preocupações similares sobre o conceito decisório. Identificado todos os ramos no mapa cognitivo, é possível criar a árvore hierárquica. Restando apenas a identificação dos pontos de vista (critérios) que farão parte do modelo multicritério com a pesquisa em cada ramo do mapa.

\subsection{Método de construção do Mapa}

O problema descrito é peculiar, pois a autoridade portuária deve cumprir obrigatoriamente o contido nas normas do ordenamento jurídico brasileiro (CF88, LF 9.966/00, LF6.938/81, 8.630/93, NBR 14253/98, NR-29 MTE, dentre outras), pelo setor ser altamente regulamentado, as concessionárias que são equiparados a servidores públicos, atuam de forma discricionária. (ANTAQ, 2009).

O facilitador pesquisou no assunto na literatura de forma a estimular o decisor (especialista em segurança do trabalho e Meio Ambiente) a considerar conceitos que representam a mens legis que são importantes ao problema, e este estabelecer a inter-relação entre estes conceitos e os seus, bem como a força das ligações 
(agrupamento). Tal procedimento foi denominado neste trabalho mapa cognitivo induzido, considerando que em setores altamente regulamentados identificou-se a necessidade desta adaptação de forma a atender plenamente o mínimo estabelecido no ordenamento jurídico.

O processo de reflexão inicial representa uma alteração ao modelo demonstrado na Figura 1, acrescentado uma entrada adicional, fornecida pelo facilitador, para estimular o decisor.

\section{RESULTADOS E DISCUSSÃO}

A partir das reflexões sobre a Estruturação de um sistema de auditoria ambiental portuária, pode-se construir o mapa cognitivo apresentado no anexo I.

Na Figura 2, apresenta-se um recorte do Mapa mostrando o resultado de um dos grupos obtidos -

Gestão Administrativa.

Figura 2 - Recorte do Mapa Cognitivo, Cluster 1 - Gestão Administrativa.

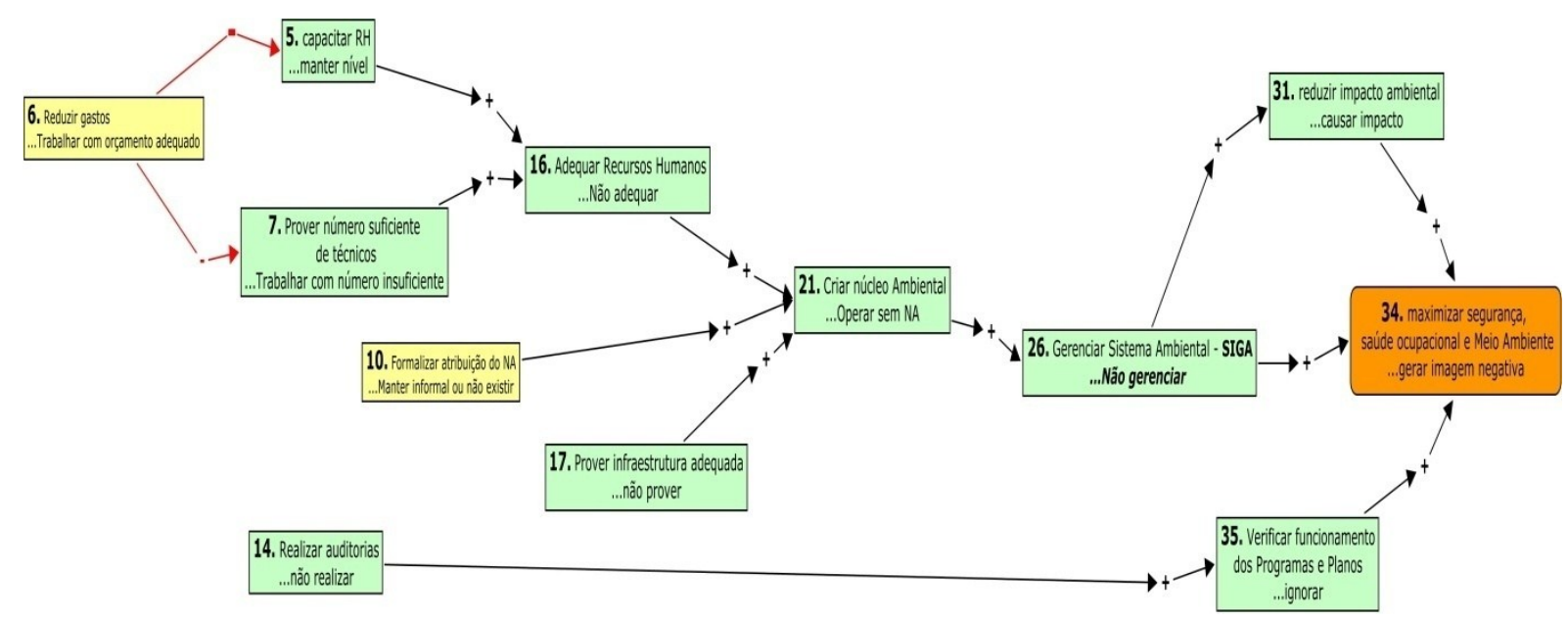

Na Tabela 1 estão relacionadas todas as linhas de argumentação, sequência de conceitos, bem como os grupos definidos pela análise do Mapa em conjunto com o Decisor. 
Tabela 1 - Detalhamento dos Grupos, Linhas de Argumentação e sequência de conceitos do Mapa Conceitual: Auditoria interna portuária com foco na Segurança Ocupacional, Saúde e Meio Ambiente.

\begin{tabular}{|c|c|c|}
\hline Grupo & $\begin{array}{c}\text { Linhas de } \\
\text { argumentação }\end{array}$ & Sequência de conceitos \\
\hline \multirow{30}{*}{ Risco Operacional } & A1 & $\mathrm{C} 1 \rightarrow \mathrm{C} 22 \rightarrow \mathrm{C} 29 \rightarrow \mathrm{C} 34$ \\
\hline & A2 & $\mathrm{C} 2 \rightarrow \mathrm{C} 11 \rightarrow \mathrm{C} 15 \rightarrow \mathrm{C} 29 \rightarrow \mathrm{C} 34$ \\
\hline & A3 & $\mathrm{C} 2 \rightarrow \mathrm{C} 11 \rightarrow \mathrm{C} 15 \rightarrow \mathrm{C} 18 \rightarrow \mathrm{C} 23 \rightarrow \mathrm{C} 30 \rightarrow \mathrm{C} 34$ \\
\hline & A4 & $\mathrm{C} 2 \rightarrow \mathrm{C} 11 \rightarrow \mathrm{C} 15 \rightarrow \mathrm{C} 31 \rightarrow \mathrm{C} 34$ \\
\hline & A5 & $\mathrm{C} 2 \rightarrow \mathrm{C} 11 \rightarrow \mathrm{C} 19 \rightarrow \mathrm{C} 30 \rightarrow \mathrm{C} 34$ \\
\hline & A6 & $\mathrm{C} 2 \rightarrow \mathrm{C} 8 \rightarrow \mathrm{C} 12 \rightarrow \mathrm{C} 11 \rightarrow \mathrm{C} 15 \rightarrow \mathrm{C} 29 \rightarrow \mathrm{C} 34$ \\
\hline & A7 & $\mathrm{C} 2 \rightarrow \mathrm{C} 8 \rightarrow \mathrm{C} 12 \rightarrow \mathrm{C} 11 \rightarrow \mathrm{C} 15 \rightarrow \mathrm{C} 18 \rightarrow \mathrm{C} 23 \rightarrow \mathrm{C} 30 \rightarrow \mathrm{C} 34$ \\
\hline & A8 & $\mathrm{C} 2 \rightarrow \mathrm{C} 8 \rightarrow \mathrm{C} 12 \rightarrow \mathrm{C} 11 \rightarrow \mathrm{C} 15 \rightarrow \mathrm{C} 31 \rightarrow \mathrm{C} 34$ \\
\hline & A9 & $\mathrm{C} 2 \rightarrow \mathrm{C} 8 \rightarrow \mathrm{C} 12 \rightarrow \mathrm{C} 11 \rightarrow \mathrm{C} 19 \rightarrow \mathrm{C} 30 \rightarrow \mathrm{C} 34$ \\
\hline & A10 & $\mathrm{C} 2 \rightarrow \mathrm{C} 8 \rightarrow \mathrm{C} 20 \rightarrow \mathrm{C} 25 \rightarrow \mathrm{C} 31 \rightarrow \mathrm{C} 34$ \\
\hline & A11 & $\mathrm{C} 2 \rightarrow \mathrm{C} 4 \rightarrow \mathrm{C} 34$ \\
\hline & A12 & $\mathrm{C} 3 \rightarrow \mathrm{C} 30 \rightarrow \mathrm{C} 34$ \\
\hline & $\overline{\mathrm{A} 13}$ & $\mathrm{C} 3 \rightarrow \mathrm{C} 24 \rightarrow \mathrm{C} 31 \rightarrow \mathrm{C} 34$ \\
\hline & A14 & $\mathrm{C} 3 \rightarrow \mathrm{C} 31 \rightarrow \mathrm{C} 34$ \\
\hline & A15 & $\mathrm{C} 3 \rightarrow \mathrm{C} 12 \rightarrow \mathrm{C} 11 \rightarrow \mathrm{C} 15 \rightarrow \mathrm{C} 29 \rightarrow \mathrm{C} 34$ \\
\hline & A16 & $\mathrm{C} 3 \rightarrow \mathrm{C} 12 \rightarrow \mathrm{C} 11 \rightarrow \mathrm{C} 15 \rightarrow \mathrm{C} 18 \rightarrow \mathrm{C} 23 \rightarrow \mathrm{C} 30 \rightarrow \mathrm{C} 34$ \\
\hline & A17 & $\mathrm{C} 3 \rightarrow \mathrm{C} 12 \rightarrow \mathrm{C} 11 \rightarrow \mathrm{C} 15 \rightarrow \mathrm{C} 31 \rightarrow \mathrm{C} 34$ \\
\hline & A18 & $\mathrm{C} 3 \rightarrow \mathrm{C} 12 \rightarrow \mathrm{C} 11 \rightarrow \mathrm{C} 19 \rightarrow \mathrm{C} 30 \rightarrow \mathrm{C} 34$ \\
\hline & A19 & $\mathrm{C} 6 \rightarrow \mathrm{C} 4 \rightarrow \mathrm{C} 34$ \\
\hline & A20 & $\mathrm{C} 6 \rightarrow \mathrm{C} 5 \rightarrow \mathrm{C} 8 \rightarrow \mathrm{C} 12 \rightarrow \mathrm{C} 11 \rightarrow \mathrm{C} 15 \rightarrow \mathrm{C} 29 \rightarrow \mathrm{C} 34$ \\
\hline & A21 & $\mathrm{C} 6 \rightarrow \mathrm{C} 5 \rightarrow \mathrm{C} 8 \rightarrow \mathrm{C} 12 \rightarrow \mathrm{C} 11 \rightarrow \mathrm{C} 15 \rightarrow \mathrm{C} 18 \rightarrow \mathrm{C} 23 \rightarrow \mathrm{C} 30 \rightarrow \mathrm{C} 34$ \\
\hline & A22 & $\mathrm{C} 6 \rightarrow \mathrm{C} 5 \rightarrow \mathrm{C} 8 \rightarrow \mathrm{C} 12 \rightarrow \mathrm{C} 11 \rightarrow \mathrm{C} 15 \rightarrow \mathrm{C} 31 \rightarrow \mathrm{C} 34$ \\
\hline & A23 & $\mathrm{C} 6 \rightarrow \mathrm{C} 5 \rightarrow \mathrm{C} 8 \rightarrow \mathrm{C} 12 \rightarrow \mathrm{C} 11 \rightarrow \mathrm{C} 19 \rightarrow \mathrm{C} 30 \rightarrow \mathrm{C} 34$ \\
\hline & A24 & $\mathrm{C} 6 \rightarrow \mathrm{C} 5 \rightarrow \mathrm{C} 8 \rightarrow \mathrm{C} 20 \rightarrow \mathrm{C} 25 \rightarrow \mathrm{C} 31 \rightarrow \mathrm{C} 34$ \\
\hline & A30 & $\mathrm{C} 6 \rightarrow \mathrm{C} 7 \rightarrow \mathrm{C} 9 \rightarrow \mathrm{C} 12 \rightarrow \mathrm{C} 11 \rightarrow \mathrm{C} 15 \rightarrow \mathrm{C} 29 \rightarrow \mathrm{C} 34$ \\
\hline & A31 & $\mathrm{C} 6 \rightarrow \mathrm{C} 7 \rightarrow \mathrm{C} 9 \rightarrow \mathrm{C} 12 \rightarrow \mathrm{C} 11 \rightarrow \mathrm{C} 15 \rightarrow \mathrm{C} 18 \rightarrow \mathrm{C} 23 \rightarrow \mathrm{C} 30 \rightarrow \mathrm{C} 34$ \\
\hline & A32 & $\mathrm{C} 6 \rightarrow \mathrm{C} 7 \rightarrow \mathrm{C} 9 \rightarrow \mathrm{C} 12 \rightarrow \mathrm{C} 11 \rightarrow \mathrm{C} 15 \rightarrow \mathrm{C} 31 \rightarrow \mathrm{C} 34$ \\
\hline & A33 & $\mathrm{C} 6 \rightarrow \mathrm{C} 7 \rightarrow \mathrm{C} 9 \rightarrow \mathrm{C} 12 \rightarrow \mathrm{C} 11 \rightarrow \mathrm{C} 19 \rightarrow \mathrm{C} 30 \rightarrow \mathrm{C} 34$ \\
\hline & A34 & $\mathrm{C} 6 \rightarrow \mathrm{C} 7 \rightarrow \mathrm{C} 9 \rightarrow \mathrm{C} 28 \rightarrow \mathrm{C} 33 \rightarrow \mathrm{C} 34$ \\
\hline & A44 & $\mathrm{C} 13 \rightarrow \mathrm{C} 32 \rightarrow \mathrm{C} 34$ \\
\hline \multirow{14}{*}{ Gestão Administrativa } & A25 & $\mathrm{C} 6 \rightarrow \mathrm{C} 5 \rightarrow \mathrm{C} 16 \rightarrow \mathrm{C} 21 \rightarrow \mathrm{C} 26 \rightarrow \mathrm{C} 31 \rightarrow \mathrm{C} 34$ \\
\hline & A26 & $\mathrm{C} 6 \rightarrow \mathrm{C} 5 \rightarrow \mathrm{C} 16 \rightarrow \mathrm{C} 21 \rightarrow \mathrm{C} 26 \rightarrow \mathrm{C} 34$ \\
\hline & A27 & $\mathrm{C} 6 \rightarrow \mathrm{C} 7 \rightarrow \mathrm{C} 16 \rightarrow \mathrm{C} 21 \rightarrow \mathrm{C} 26 \rightarrow \mathrm{C} 31 \rightarrow \mathrm{C} 34$ \\
\hline & A28 & $\mathrm{C} 6 \rightarrow \mathrm{C} 7 \rightarrow \mathrm{C} 16 \rightarrow \mathrm{C} 21 \rightarrow \mathrm{C} 26 \rightarrow \mathrm{C} 34$ \\
\hline & A29 & $\mathrm{C} 6 \rightarrow \mathrm{C} 7 \rightarrow \mathrm{C} 16 \rightarrow \mathrm{C} 21 \rightarrow \mathrm{C} 27 \rightarrow \mathrm{C} 31 \rightarrow \mathrm{C} 34$ \\
\hline & A35 & $\mathrm{C} 6 \rightarrow \mathrm{C} 17 \rightarrow \mathrm{C} 21 \rightarrow \mathrm{C} 26 \rightarrow \mathrm{C} 31 \rightarrow \mathrm{C} 34$ \\
\hline & A36 & $\mathrm{C} 6 \rightarrow \mathrm{C} 17 \rightarrow \mathrm{C} 21 \rightarrow \mathrm{C} 26 \rightarrow \mathrm{C} 34$ \\
\hline & A37 & $\mathrm{C} 6 \rightarrow \mathrm{C} 5 \rightarrow \mathrm{C} 16 \rightarrow \mathrm{C} 21 \rightarrow \mathrm{C} 27 \rightarrow \mathrm{C} 31 \rightarrow \mathrm{C} 34$ \\
\hline & A38 & $\mathrm{C} 14 \rightarrow \mathrm{C} 35 \rightarrow \mathrm{C} 34$ \\
\hline & A39 & $\mathrm{C} 14 \rightarrow \mathrm{C} 26 \rightarrow \mathrm{C} 31 \rightarrow \mathrm{C} 34$ \\
\hline & A40 & $\mathrm{C} 14 \rightarrow \mathrm{C} 26 \rightarrow \mathrm{C} 34$ \\
\hline & A41 & $\mathrm{C} 10 \rightarrow \mathrm{C} 21 \rightarrow \mathrm{C} 26 \rightarrow \mathrm{C} 31 \rightarrow \mathrm{C} 34$ \\
\hline & A42 & $\mathrm{C} 10 \rightarrow \mathrm{C} 21 \rightarrow \mathrm{C} 26 \rightarrow \mathrm{C} 34$ \\
\hline & A43 & $\mathrm{C} 10 \rightarrow \mathrm{C} 21 \rightarrow \mathrm{C} 27 \rightarrow \mathrm{C} 31 \rightarrow \mathrm{C} 34$ \\
\hline
\end{tabular}

\section{É importante ressaltar que a análise do Mapa deixa claro um paradoxo} representado pelas ligações de influência negativas (representadas por setas Revista Produção Online. Florianópolis, SC, v.11, n. 4, p. 950-964, out./dez. 2011. 
vermelhas), que vem ao encontro da lógica Proteção x Produção, ou seja, a Redução de Gastos no cluster 1 - Gestão Administrativa faz parte da problemática da auditoria interna portuária, isto é trabalhar com orçamento adequado é um ponto importante para o processo. Da mesma forma é o conceito "Sofrer pressão por prazos", que também influencia negativamente.

É possível notar que dada a complexidade do constructo, muitas linhas de argumentação foram geradas, possibilitando diferentes caminhos para o atendimento ao objetivo fundamental-estratégico identificado - Maximizar Segurança Ocupacional, Saúde e Meio Ambiente.

As definições dos pólos opostos psicológicos foram de fundamental importância para o Decisor e também para o facilitador, no sentido de melhor entender o constructo do Decisor.

Pode-se verificar, por exemplo, para o objetivo fundamental-estratégico, que seu pólo oposto é "gerar imagem negativa", ou seja, o oposto a maximizar, representa que a ocorrência de acidentes ocupacional, doenças, vazamento incidentais concorrem para promover uma imagem negativa do Porto perante a Sociedade na visão do Decisor.

A partir do Mapa cognitivo, juntamente com o Decisor, foi possível transformar o mesmo em uma estrutura hierárquica que possibilitará em situações futuras a utilização de um método multicritério para apoio à decisão. (ENSSLIN; MONTIBELLER; NORONHA, 2001).

A estrutura obtida está representada na Figura 3, com os Pontos de Vista Fundamentais elencados na primeira linha após os Clusters e Os Pontos de vista Elementares na segunda. 
Figura 3 - Estrutura Hierárquica, com Grupos, Pontos de Vista Fundamentais e Elementares.

\section{Desempenho Portuário}

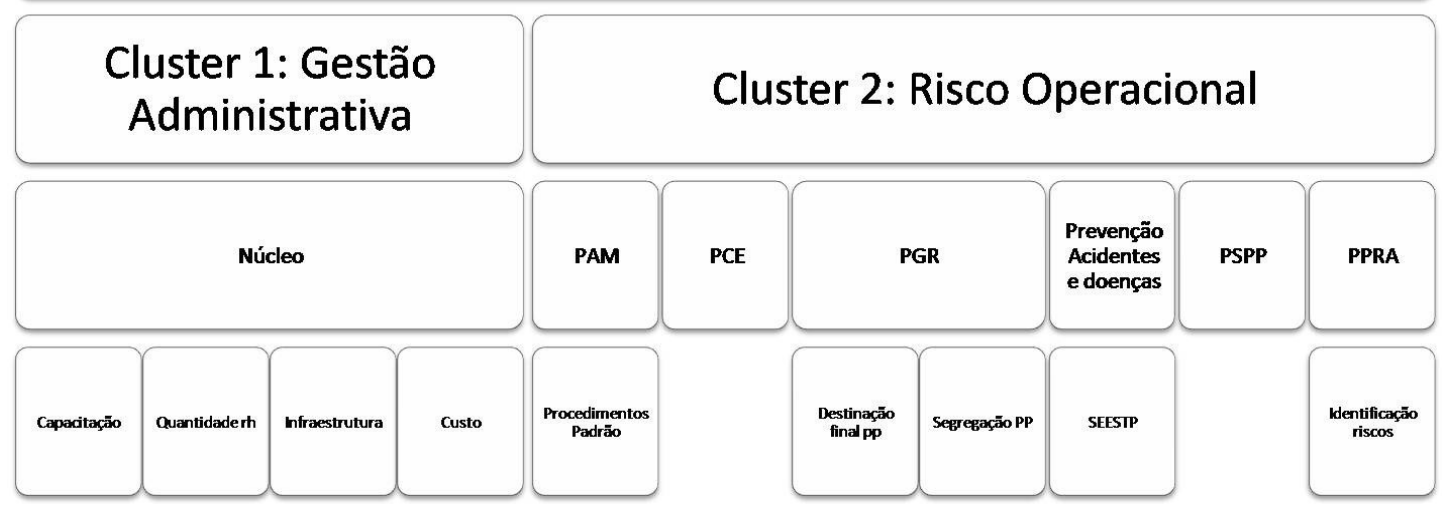

A definição dos PVF foi baseada no enquadramento proposto por KEENEY, (1992), conforme Figura 4.

Figura 4 - Quadro referência do Processo Decisório

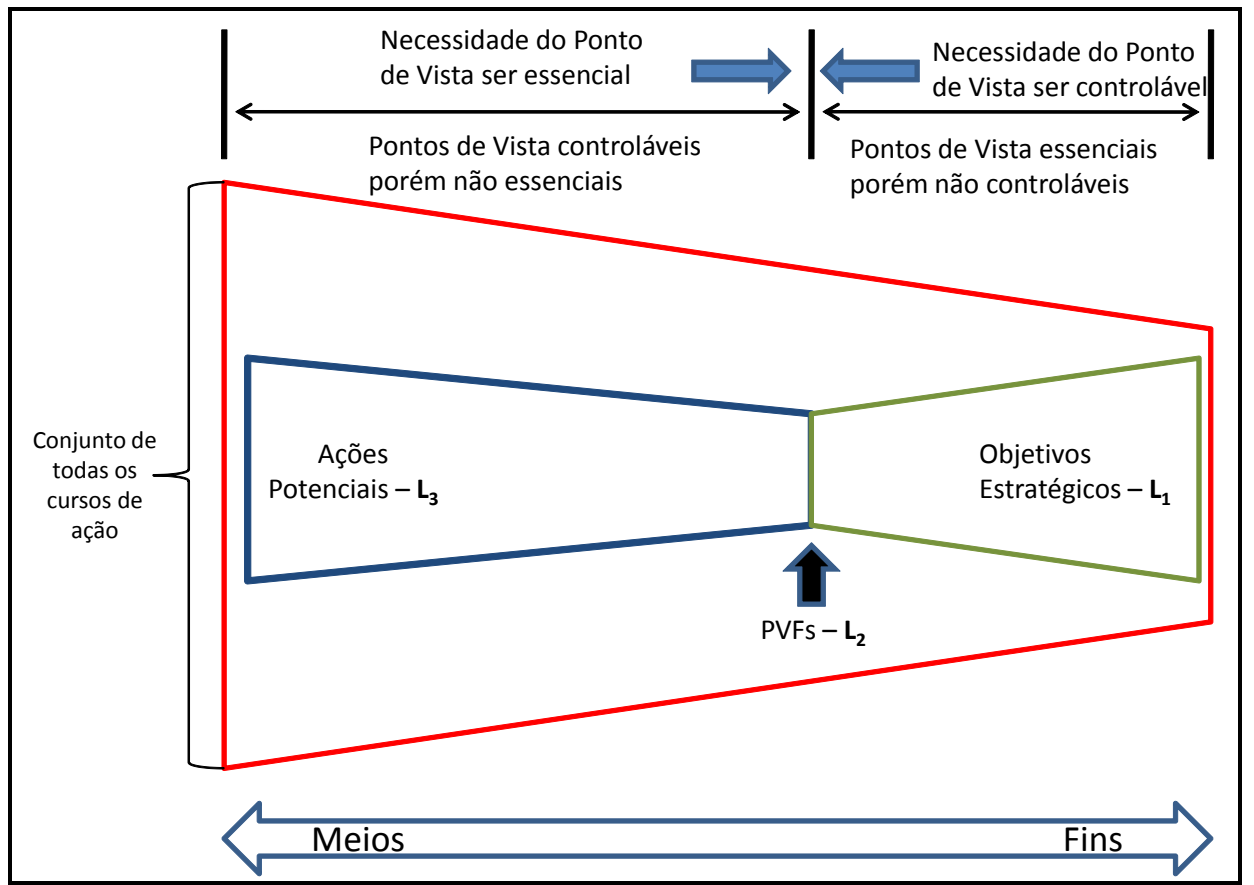

Fonte : Adaptado de KEENEY (1992).

Para o processo de obtenção dos candidatos a PVF, é necessário verificar a essenciabilidade e controlabilidade do mesmo, tendo como referência os objetivos

Revista Produção Online. Florianópolis, SC, v.11, n. 4, p. 950-964, out./dez. 2011. 
estratégicos (L1) e ações potenciais (L3). Na Figura 5 é demonstrada, a título de exemplo a obtenção do PVF "Elaborar PPRA".

Figura 5 - Exemplo de enquadramento para o PVF Elaborar PPRA, no grupo Risco Operacional.

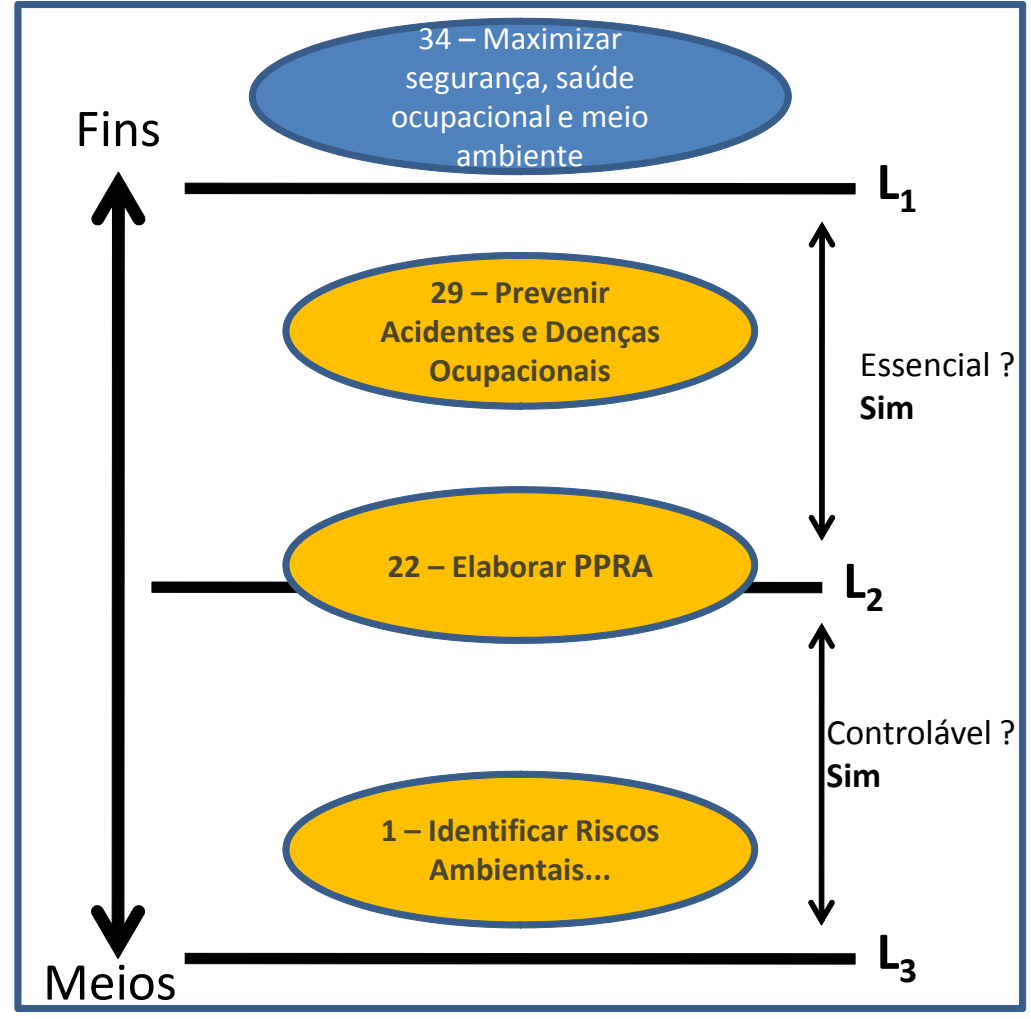

A determinação dos candidatos a PVF foi realizada como descrito acima com base no Mapa Cognitivo e na presença do Decisor, no entanto os PVF precisam ainda ser submetidos a alguns testes para verificação se os mesmos atendem as propriedades, que são: essenciabilidade, controlabilidade, ser completo, ser mensurável, ser operacional, ser isolável, não redundante, conciso e compreensível.

A verificação foi realizada para cada um dos PVF, tendo como base o mapa cognitivo, seus ramos e linhas de argumentação.

Para que um PVF seja essencial, o mesmo deve levar em conta aspectos de fundamental importância ao decisor.

O PVF deve ser controlável, isto é cada uma dos aspectos deve ser influenciado apenas pelas ações potenciais. Devem ser completos, incluindo todos os aspectos considerados como fundamentais para o decisor, mensuráveis, na medida em que é possível especificar o desempenho das ações potenciais. A Revista Produção Online. Florianópolis, SC, v.11, n. 4, p. 950-964, out./dez. 2011. 
questão da operacionabilidade, permitir que se conheçam os valores, quantidades para cada ação potencial. Devem ser isoláveis, isto é analisar um aspecto por vez, da mesma forma ser não redundante, não considerar um aspecto mais de uma vez. Ser conciso, ou seja, possuir o número mínimo para permitir modelar de forma adequada o problema, e finalmente compreensível, de significado claro para o decisor. (ENSSLIN, MONTIBELLER, NORONHA, 2001).

Cada um dos PVF foi analisado em conjunto com o Decisor, e concluiu-se que todos atendem às propriedades desejadas, conforme Tabela 2.

Tabela 2 - Resumo da avaliação dos PVF, segundo as propriedades.

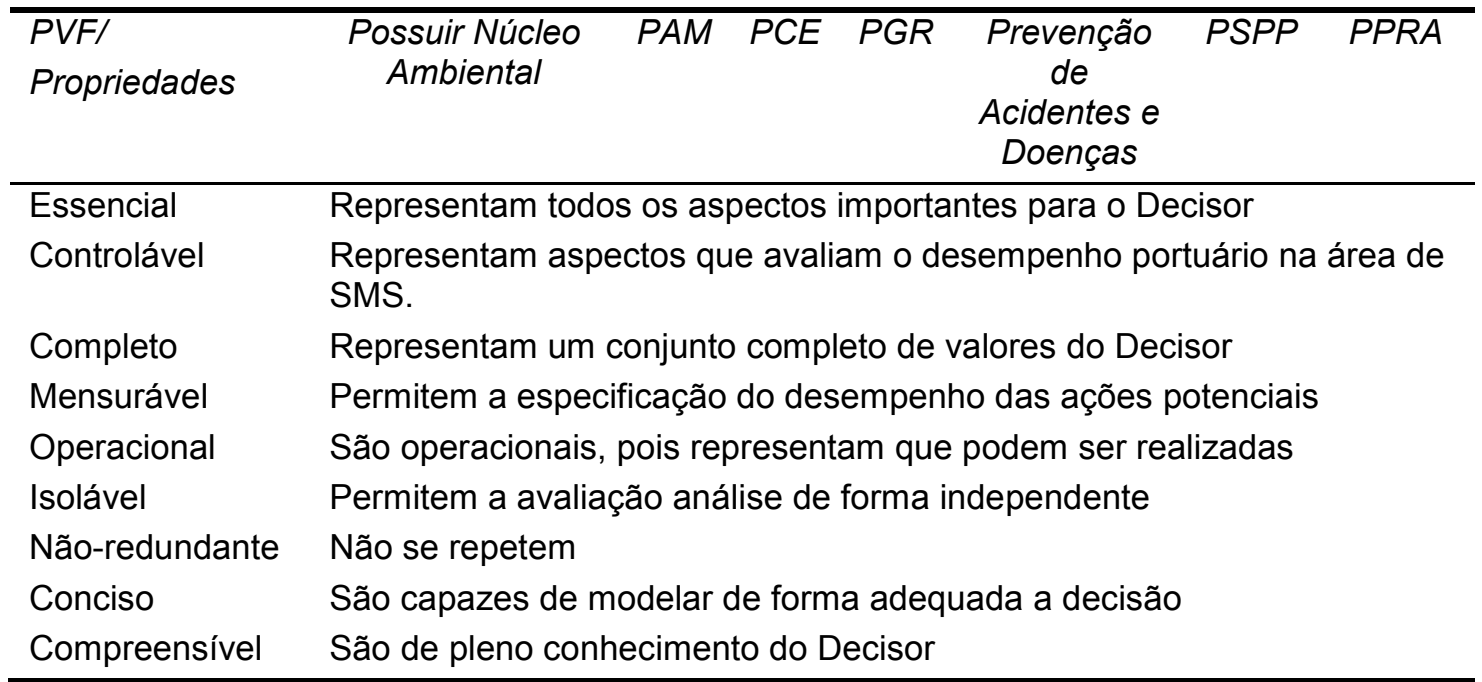

\section{CONCLUSÕES}

A construção do Mapa Cognitivo - induzido pelo facilitador, visando garantir o atendimento à legislação específica da área portuária mostrou-se satisfatória, pois conforme depoimento do Decisor: "A questão do Plano de Segurança Pública Portuária, apesar de em alguns momentos envolver questões de atendimento à emergência, talvez não fosse considerada no Mapa, em função da formação do profissional, assim destaco que é importante para o Decisor receber informações pertinentes sobre o problema em análise"

Como se trata de um problema complexo, conforme citado anteriormente, a passagem do Mapa Cognitivo para um Diagrama Hierárquico não é trivial, sendo necessárias várias reuniões facilitador-decisor a construção do mesmo.

Revista Produção Online. Florianópolis, SC, v.11, n. 4, p. 950-964, out./dez. 2011. 
A obtenção dos PVF possibilitará a construção de modelos multicritério de apoio à decisão, que os autores sugerem como continuidade deste trabalho a elaboração dos descritores e aplicação de um método multicritério, estabelecendo uma avaliação do desempenho portuário sob a ótica ambiental.

\section{AGRADECIMENTOS}

Os autores agradecem as sugestões dos revisores, que colaboraram no aperfeiçoamento deste artigo.

\section{REFERÊNCIAS}

ANTAQ - Agência Nacional de Transporte Aquaviário. 2009. Acesso em: 15 out. 2009. Disponível em: http://www.antaq.gov.br/portal/default.asp.

BRASIL. Conselho Nacional do Meio Ambiente (CONAMA). Resolução do CONAMA $n^{\circ} 237$, de 19 de dezembro de 1997 - Regulamenta os aspectos de licenciamento ambiental estabelecidos na Política Nacional do Meio Ambiente. DOU $n^{\circ} 247$, de 22/12/1997, p. 30.841-30.843

BRASIL. Conselho Nacional do Meio Ambiente (CONAMA). Resolução do CONAMA $n^{\circ}$ 237, de 19 de dezembro de 1997. Estabelece os requisitos mínimos e o termo de referência para realização de auditorias ambientais. DOU $n^{\circ} 138$, de 05/07/2002, p. $75-76$

CAIXETA-FILHO J. V. Gestão logística do transporte de cargas. São Paulo: Atlas, 2001.

DINIZ M E e LINS M. P.E. Estruturação de Problemas Sociais Complexos utilizando Mapas Cognitivos. In: ENCONTRO NACIONAL DE ENGENHARIA DE PRODUÇÃO, 24, 2009. Anais... Salvador: ENEGEP, 2009.

ENSSLIN S.; ENSSLIN, L.; DUTRA, A.; IGARASHI, D.C.C. Visão Hard ou visão Soft da Pesquisa Operacional? Reflexões sobre posturas e procedimentos. In: SIMPÓSIO DE ENGENHARIA DE PRODUÇÃO, 12, 2005. Anais ... Bauru: SIMPEP, 2005.

ENSSLIN L., MONTIBELLER G. N.; NORONHA S. M. Apoio à decisão: metodologias para estruturação de problemas e avaliação multicritério de alternativas. Florianópolis : Insular, 2001.

KEENEY R. L. Value-focused thinking: a path to creative Decision-making. Cambridge : Harvard Univ. Press, 1992. 
MINGERS J. e ROSENHEAD J. Problem Structuring Methods in Action. European Journal of Operation Research , v. 152, p.530 - 554, 2004.

ROSENHEAD J. e MINGERS J. Rational analysis for a problematic world Revisited. New York : John Wiley \& Sons, 2001.

VIASEG. 2008. Seg.Trabalho : acidentes no Porto do ES. Acesso em: 12 nov. 2009. Disponível em: <http://www.viaseg.com.br/noticia/6329-

segtrabalho acidentes no porto do es.html>

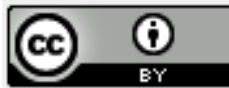

Artigo recebido em 05/04/2010 e aceito para publicação em 11/06/2011.

Revista Produção Online. Florianópolis, SC, v.11, n. 4, p. 950-964, out./dez. 2011. 
Anexo I: Mapa Cognitivo completo

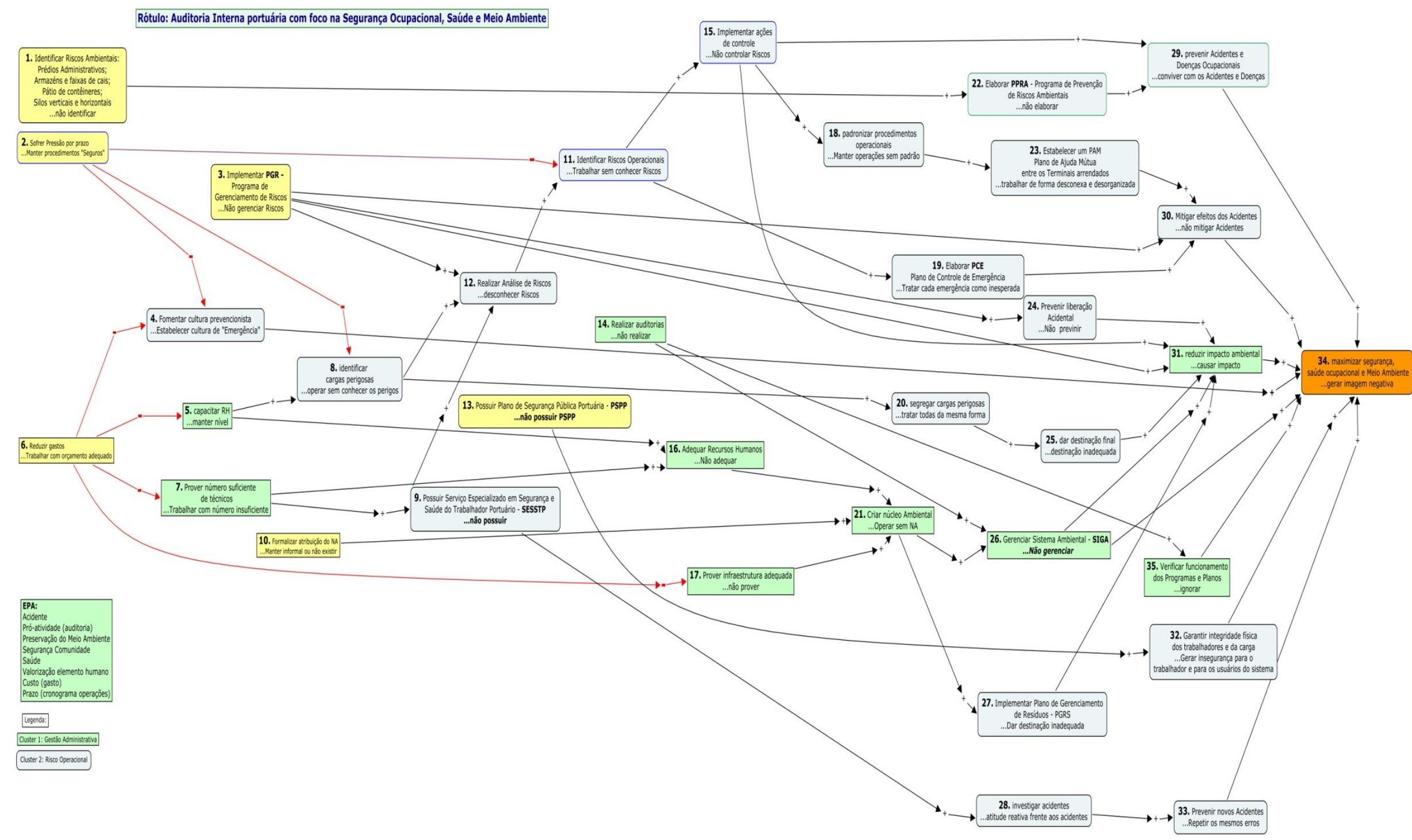

Revista Produção Online. Florianópolis, SC, v.11, n. 4, p. 950-964, out./dez. 2011. 